\title{
New techniques for removing static shift from CSAMT data based on Fourier transform and Radon transform
}

\author{
Xiaodong Luan, Qingyun Di \\ Key Lab of Shale gas and Geoengineering, Institute of \\ Geology and Geophysics \\ Chinese Academy of Sciences \\ Beijing, China
}

\author{
Xiaodong Luan \\ University of Chinese Academy of Sciences \\ Beijing, China
}

\begin{abstract}
This paper proposed two new techniques to reduce the static shift effect in Controlled Source Audio-frequency Magnetotelluric (CSAMT) method in terms of image and signal, by applying Radon transform and Fourier transform, respectively, for data processing. The first method compensates for the static effect in CSAMT data by repositioning the value
\end{abstract}

around $\theta=90^{\circ}$ and $\theta=270^{\circ}$ in Radon domain. The second

method corrects the CSAMT data by smoothening out the lowfrequency components, in Fourier domain. We established 3D model to simulate the static shift that occurs when a low resistive body exists near the surface and corrects it with the two methods. We also applied the two methods to real CSAMT data. The study proved that the effects of small-scale scattering caused by inhomogeneity near the surface can be effectively removed by Radon transform method and Fourier transform method.

Keywords-Fourier tranform; Radon transform; CSAMT; static shift

\section{INTRODUCTION}

CSAMT is an artificial source electromagnetic sounding method. Compared to Magnetotelluric method(MT) and Audio-frequency Magnetotelluric method(AMT), CSAMT is more efficient and has better SNR. Therefore, it has been widely used in mineral exploration, geothermal exploration, and groundwater exploration studies ${ }^{[1]}$. However, similar to MT and AMT, shallow inhomogeneous bodies can lead to inaccurate interpretation of CSAMT data by shifting the CSAMT apparent resistivity sounding curve by a factor, which is independent of the survey frequency. This phenomenon is called static shift and it could cause false steep faults in CSAMT data interpretation. The amount of parallel shift, commonly referred to as the CSAMT static shift, cannot be determined directly from conventionally recorded CSAMT data at a single site. Since 1970s, Lots of experts have done researches on static shift and have provided so many methods to correct static shift, such as EMAP method ${ }^{[2]}$, TEM method ${ }^{[3]}$, phase method ${ }^{[4]}$, Wavelet method ${ }^{[5]}$, inversion method ${ }^{[6][7]}$ and so on. Nevertheless, these methods cannot completely remove the static shift. Therefore, we proposed two methods, Radon transform method and Fourier method, which can efficiently remove the static effect on CSAMT data.

\section{RADON TRANSFORM}

In 1917, Austrian mathematician, J. Radon, proved that one function can be reestablished from its "projection" in Euclidean $2 \mathrm{D}$ or $3 \mathrm{D}$ space and that there exists only one inversion for total projection function. This has since been established as the mathematical foundation for tomography and image reconstruction. Since 1970 s, the theory and application of Radon transform have been booming and have been widely used in physics, medical science, astronomy, materialogy, geophysics and so on.

Forward transformation of Radon ${ }^{[8]}$ : for two-dimensional function $\mathrm{f}(\mathrm{x}, \mathrm{y})$, the Radon transform $\operatorname{Rf}(\mathrm{p}, \theta)$ is defined as the integration along line $l$, which is determined by the $\mathrm{p}$ and $\theta$.

$$
\begin{aligned}
& R_{f}(\mathrm{p}, \theta)=\int_{-\infty}^{\infty} f(x, y) d l \\
& \text { * MERGEFORMAT (1) }
\end{aligned}
$$

Where $\mathrm{p}$ is the distance from origin and $\theta$ is angle.

The function of line 1 is $p=x \cos \theta+y \sin \theta$. Then, according to equation (1), under a specified angle, the Radon transform $\operatorname{R\theta }(\mathrm{p})$ is as follow:

$$
R_{\theta}(p)=\int_{-\infty}^{\infty} f(p \cos \theta-s \sin \theta, p \cos \theta+s \sin \theta) \mathrm{d} s
$$

\section{* MERGEFORMAT (2)}

Where, $\left[\begin{array}{l}p \\ s\end{array}\right]=\left[\begin{array}{rc}\cos \theta & \sin \theta \\ -\sin \theta & \cos \theta\end{array}\right]\left[\begin{array}{l}x \\ y\end{array}\right]$

Inverse transformation of Radon ${ }^{[9]}$ : Radon inverse transformation can be derived from two-dimensional inverse Fourier transform. For function $\mathrm{f}(\mathrm{x}, \mathrm{y})$, the inverse Fourier transform is given as: 


$$
f(\mathrm{x}, \mathrm{y})=\frac{1}{(2 \pi)^{2}} \int_{R^{2}} e^{i(\mathrm{xu}+\mathrm{vy})} F(\mathrm{u}, \mathrm{v}) d u d v
$$

।* MERGEFORMAT (3)

We assume, $\left\{\begin{array}{l}X=(x, y) \\ u=r \cos \theta, v=r \sin \theta \\ \omega=(\cos \theta, \sin \theta)\end{array}\right.$,then

$$
\begin{gathered}
f(\mathrm{X})=\frac{1}{(2 \pi)^{2}} \int_{0}^{\pi} \int_{-\infty}^{\infty} e^{i r<X, \omega>} F_{t}[\mathrm{R}(\mathrm{r}, \omega)]|r| d r d \omega \\
\quad * \text { MERGEFORMAT (4) }
\end{gathered}
$$

Equation (4) is the inversion of Radon transform.

When low-resistive anomalous bodies exist within the near surface region, the apparent resistivity curve moves either up or down on the apparent resistivity versus frequency plot, which looks like straight lines on the pseudo-section. In Radon

transform, the value of $\theta$ is set between $0^{\circ}$ and $360^{\circ}$. When

CSAMT data is transformed into Radon domain, the static shift becomes noticeable as spots near $\left(\mathrm{p}=0, \theta=90^{\circ}\right)$ and $(\mathrm{p}=0, \theta=270$ $\left.{ }^{\circ}\right)$, which is easy to correct. Then, the corrected CSAMT data is obtained through the inverse Radon transform.

\section{FOURIER TRANSFORM}

Fourier transform is an important tool in digital signal processing. The theory of Fourier transform indicates that at any given time, a serial signal is the infinite superposition of sine waves with different frequencies. Mathematically, Fourier transform is a special integral transform which can change the given function into a linear combination of cosine based function.

CSAMT is a frequency domain electromagnetic method, whose data is plotted as apparent resistivity versus frequency curve. However, from the point of view of a signal, the apparent resistivity versus frequency curve is just a special "signal", while the static shift caused by low resistive bodies is the change of "DC" component. Based on the statement above, we proposed the use of Fourier transform method to remove the static shift. First, using cubic spline, we turned the logarithmic resistivity into uniform sampling "signal". Then, the obtained signal undergoes a Fourier transformation process. After the Fourier transform, the "DC" component curve can be drawn with data from every sounding point. The "DC" component of sounding points affected by static shift will be lower or higher than others.

Second, the Chebyshev I high-pass filter ${ }^{[10][11]}$ is used to filter out the low frequency information from the "DC" component curve. The relationship between amplitude and frequency of Chebyshev I filter is as follow:

$$
\begin{gathered}
G_{n}(\omega)=\left|H_{n}(\mathrm{j} \omega)\right|=\frac{1}{\sqrt{1+\kappa^{2} T_{n}^{2}\left(\frac{\omega}{\omega_{0}}\right)}} \\
\text { * MERGEFORMAT (5) }^{*}
\end{gathered}
$$

Where, $|\kappa|<1$ is the fluctuate coefficient, $\omega 0$ is the cut-off

frequency. $T_{n}\left(\frac{\omega}{\omega_{0}}\right)$ is the Chebyshev polynomials.

Finally, we adopted the Savitzky-Golay filter ${ }^{[2]}$ to smooth the other frequency components laterally in Fourier domain. As mentioned, owing to the similarity of apparent resistivity, it is rational to smooth in Fourier domain.

\section{Simulation Result}

In order to verify the validity of Radon transform method and Fourier transform method, we designed a model with lowresistive body within the shallow subsurface region (Fig. 1). In homogeneous half space, we designed a survey line from $1000 \mathrm{~m}$ to $1000 \mathrm{~m}, 10 \mathrm{~km}$ away from the transmitter. Beneath the $0 \mathrm{~m}$, there was a low-resistive body at $2 \mathrm{~m}$ depth with size $4 \mathrm{~m} * 4 \mathrm{~m} * 2 \mathrm{~m}$ and a big conductive body at $500 \mathrm{~m}$ depth, having the size of $1000 \mathrm{~m}^{*} 1000 \mathrm{~m} * 200 \mathrm{~m}$. The dipole distance $A B$ was $1000 \mathrm{~m}$, transmitter current of $10 \mathrm{~A}$ was used, as well as frequency ranging from $20 \sim 213 \mathrm{~Hz}$ and station separation of $20 \mathrm{~m}$. The simulation result in Fig. 2 showed that the apparent resistivity at $0 \mathrm{~m}$ is much higher than the others due to the static shift.

Fig.3 shows both the correction result after Radon transform method and Fourier transform method. It is obvious that these two method can remove the static shift completely.

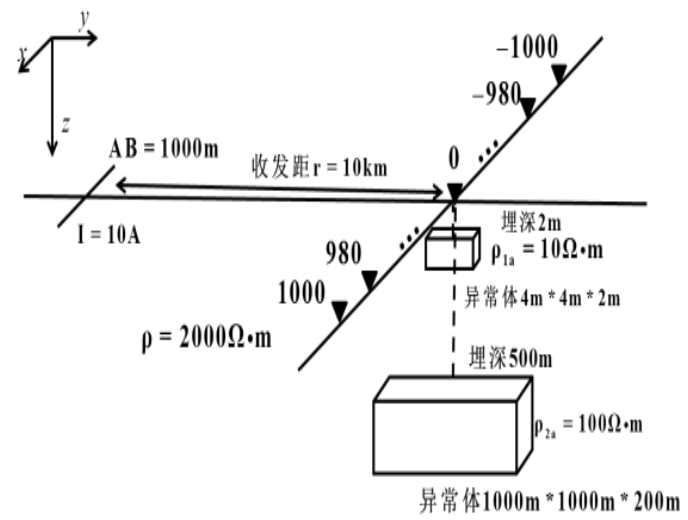

Fig.1 Forward model 


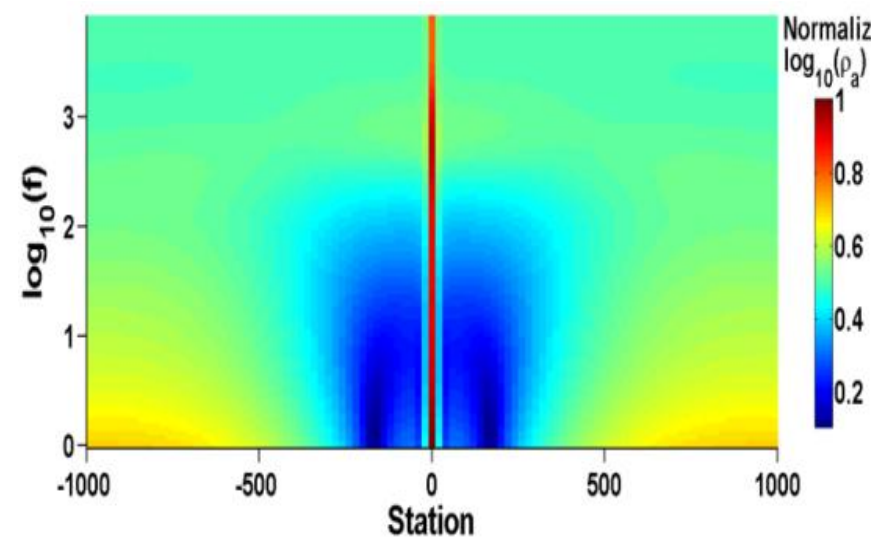

Fig.2 apparent resistivity pseudo-section

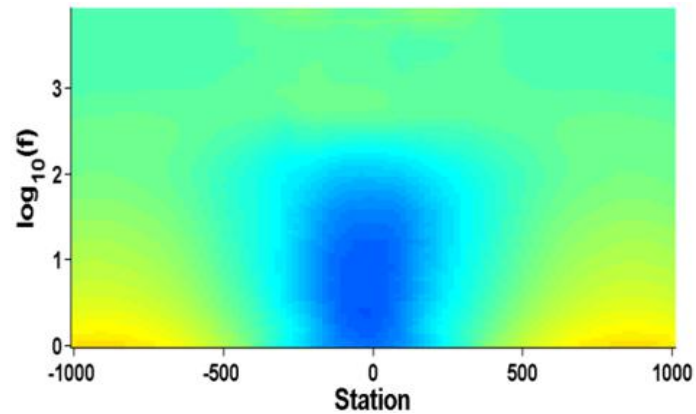

(a)

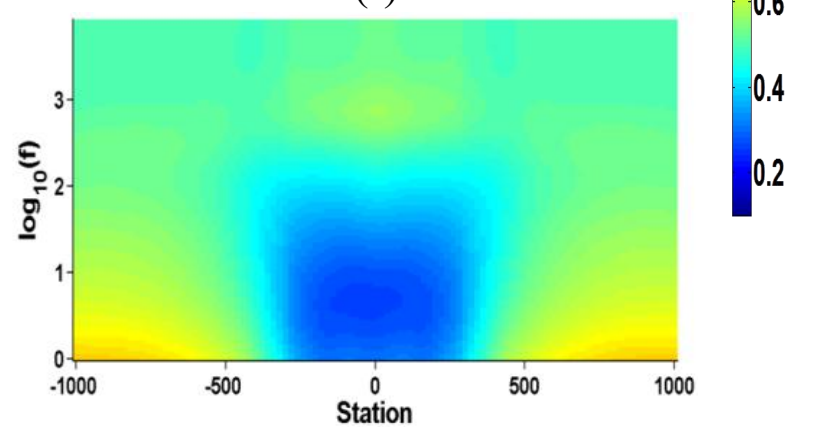

(b)

Fig. 3 The correction results

(a) Radon transform, (b) Fourier transform

\section{REAL DATA RESULT}

We chose the CSAMT data acquired in Inner-Mongolia to test the two methods. The dipole distance $\mathrm{AB}$ was $1.5 \mathrm{~km}$, the transmitter-receiver distance $\mathrm{r}$ equal $9 \mathrm{~km}$ and the transmitted frequency ranged from $1 \mathrm{~Hz}$ to $9600 \mathrm{~Hz}$. The total survey length was $4.8 \mathrm{~km}$, with station separation of $20 \mathrm{~m}$. The obtained apparent resistivity pseudo-section is shown in Figure 4(a). In order to compare the processed results with the unprocessed result, we normalized the apparent resistivity. From Figure 4(a), we could observe the static shift effect near distances $750 \mathrm{~m}$, $2500 \mathrm{~m}$ and $3000 \mathrm{~m}$, based on the fact that their apparent resistivity values are much higher than the others. We used Fourier transform method, Radon transform method and EMAP method to process the real data and the results are shown in Fig.4(b), (c) and (d). Both Radon transform method and Fourier transform method were able to eliminate the static shift, while the EMAP could not remove the static shift thoroughly, especially the small ones near $750 \mathrm{~m}$.
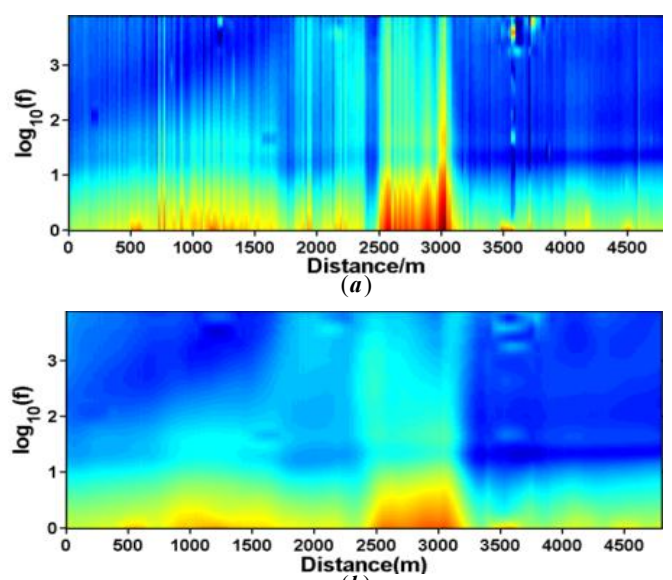

Normalized $\log _{10}(\rho)$

1

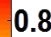

(b)

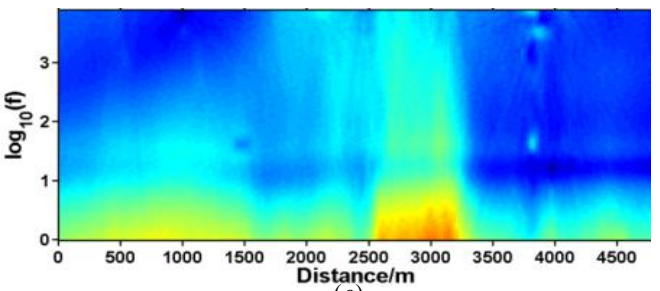

$(c)$

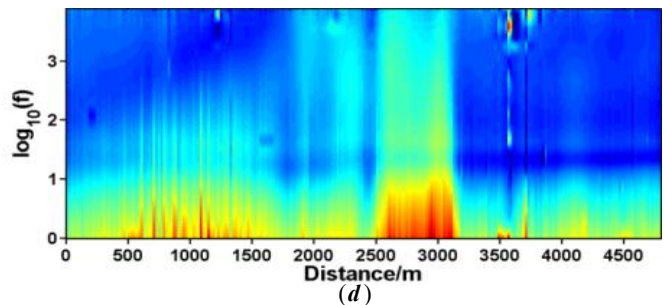

Fig. 4 Real data result

(a) Apparent resistivity pseudo-section, (b) Fourier transform method, (c) Radon transform method, (d) EMAP

\section{CONCLUSION}

The Radon transform method and Fourier transform method were effective in removing the static shift due to localized shallow inhomogeneity. The results obtained from both the simulation and real data, shows that these two techniques can remove the static shift from CSAMT data without affecting the response from deep structures.

\section{ACKNOWLEDGMENT}

The authors appreciate Dr. Fayemi for helping revise the paper and thank the other research group members.

\section{REFERENCES}

[1] He J S. 1990, Controlled source audio frequency magnetotelluric method. South China Industrial University Press.

[2] Verdin. C.T.,1991,Continuous profiling of magnetotelluric Fields, D. thesis, University of California. 
[3] Ben K. Sternberg, James C, Louise Pellerin. 1988.Correction for the static shift in magnetotellurics using transient electromagnetic soundings Geophysics, 53(11), 1459-1468

[4] Shi X X, Yan S, Chen M S,et al. 2011, Correction of CSAMT static shift using transformed phase, Oil geophysical prospecting, 46(5), 802-809.

[5] Song S G, Tang J T, He J S, 1995, Wavelet analysis and the recognition, separation and removal of the static shift in electromagnetic sounding. Chese J Geophys, 38(1):120 128.

[6] De Groot-Hedlin C ,1991, Removal of static shift in two dimensions by regularized inversion. GeoPhysies, 56: 2102 2106.

[7] Ogawa Y, Uchia T.A, 1996, Two-dimension MT inversion assuming static shift, Geophysical Journal international, 126:69-76
[8] Gregory B. 1987. Discrete radon transform. IEEE transactions on acoustics,speech and signal processing. ASSP-35(2), 162-172.

[9] W.R. Madych. 2004. Radon's inversion foumulas. Transaction of the American mathematical society, 356(11),4475-4491.

[10] John P. Boyd, 1991, Chebyshev and Fourier spectral method. Dover Publication, Inc.

[11] Richard J.C, 1999, General coupling matrix synthesis method for Chebyshev filtering functions. IEEE transactions on microwave theory and techniques, 47(4):433-442.

[12] Jianwen Luo, Kui Ying, Jing Bai. 2005, Savitzky-Golay smoothing and differentiation filter for even number data. Signal processing, 85: 14291434 